\title{
A kinetic study of the lipase-catalyzed ethanolysis of two short chain triradylglycerols: alkylglycerols vs. triacylglycerols
}

Luis Vázquez ${ }^{1}$, Oscar Fernandez ${ }^{1}$, Rosa M. Blanco ${ }^{2}$, F. Javier Señoráns ${ }^{1}$, Guillermo Reglero ${ }^{1}$, and Carlos F. Torres ${ }^{1 *}$

${ }^{1}$ Sección Departamental Ciencias de la Alimentación, Facultad de Ciencias, Universidad Autónoma de Madrid, 28049 Cantoblanco, Madrid, Spain

2Instituto de Catálisis y Petroleoquímica. CSIC. Marie Curie, 2, 28049 Madrid, Spain

${ }^{*}$ Corresponding author

Telephone: +34914973091

Fax: + 34914978255

E-mail: carlos.torres@uam.es 


\section{Abstract}

Lipase-catalyzed ethanolysis of two short-chain triradylglycerols, namely tributyrin and 2,3-dibutyroil-1-O-alkylglycerols, have been studied. Much faster rate of reaction for the ethanolysis of tributyrin than that of 2,3 dibutyroil-1-O-alkylglycerols was attained. A kinetic model for the rate of release of ethyl butyrate and for the inactivation of the lipase has been also studied. The parameter corresponding to the release of ethyl butyrate was one order of magnitude higher for ethanolysis of tributyrin than the corresponding of 2,3-dibutyroil-1-O-alkylglycerols.

On the contrary, the stability of Novozym 435 during ethanolysis of 2,3dibutyroil-1-O-alkylglycerols was higher than the corresponding of tributyrin.

At the reaction conditions under study, both ethanolysis reactions take place with high selectivity and yield monoesterified alkylglycerols and sn-2 monobutyrin as the main acylglycerols in the reaction mixtures.

Keywords: Candida antarctica, alkylglycerols, ethanolysis, butyric, lipase, kinetics model.

\section{Introduction}

Alkylglycerols, alkylglycerophospholipids and their derivatives, commonly known as ether lipids, have been the subject of much attention because of their special physiological functions in humans [1, 2]. Ether lipids have been used in the therapy of cancer [3], since they are potent antineoplastic agents which inhibit growth, show antimetastatic activity and induce differentiation and apoptosis in cancer cells [4, 5]. Immune stimulators 
properties have also been attributed to dietary ingestion of these substances [6].

In recent years, numerous synthetic ether lipids that do not occur in nature have been prepared to find therapeutic agents such as agonists and antagonists of natural lipid mediators [7]. One example of the mentioned synthetic ether lipids are PAF mimics that retain a short-chain residue esterified at the $s n-2$ position [8].

The studies done during the last decade provide multiple lines of evidence that butyrate indeed interferes with the pathogenesis of colorectal cancer. Butyrate inhibits DNA synthesis and arrests growth of neoplastic colonocytes [9], modifies expression of genes involved in chemotherapy resistance [10] and in cell proliferation/differentiation [11,12], and induces apoptosis [13]. At least some of butyrate's antineoplastic effects in colon cancer cells may be due to its synergistic action with another antiproliferative agent, 1,25-dihydroxyvitamin D3 [dihydroxycholecalciferol; $(\mathrm{OH}) 2 \mathrm{D} 3]$. In various cancer cell lines it has been shown that butyrate and $(\mathrm{OH}) 2 \mathrm{D} 3$ act synergistically in reducing proliferation and enhancing differentiation of neoplastic cells $[14,15]$.

In spite of its early promise, butyrate is not among the drugs used for cancer treatment. The major problem has been to achieve and maintain its millimolar concentrations in blood. Butyrate is metabolized rapidly as soon as it enters the colonocyte via its active transport system $[16,17,18]$, and its plasma concentrations are far below those required to exert its antiproliferative/differentiating actions. Prodrug of natural butyrate, such as tributyrin (TB) and 2,3-dibutyroil-1-O-alkylglycerols (SCAKG), are neutral short-chain fatty acid triradylglycerols that are likely to overcome the pharmacokinetic drawbacks of natural butyrate as a drug [19]. 
In addition, recent studies indicate that butyrate and fish oil work coordinately to protect against colon tumorigenesis [20, 21] Based on these findings, designing new lipidic vehicles structures in which these two fatty acids are incorporated into the body could influence the location of their release and it may allow targeting of these bioactive molecules to specific areas of other tissues.

One of the problems that arise is to elucidate which of these two lipidic vehicles, namely alkylglycerols and triacylglycerols, is more efficient and chemically stable in plasma, diffuses through biological membranes and is metabolized by intracellular lipases, releasing therapeutically effective fatty acids over time directly into the cell.

In a first attempt, the present study focuses on the kinetics of the lipase catalyzed ethanolysis of these two lipidic vehicles containing butyric acid residues. The results obtained fulfill two objectives: 1) show differences in rate of the reaction, selectivity, and provide mechanistic information regarding how these two compounds are recognized by lipases, and 2) lipase-catalyzed ethanolysis of these two triradylglycerol is very useful step for selectively remove fatty acid residues at sn-1, and sn-3 positions and it can be used for subsequent production of structured lipids containing short chain and polyunsaturated fatty acids in a single molecule.

\section{Experimental}

\subsection{Materials}

2,3-dibutyroil-1-O-octadecylglycerol (SCAKG) was synthesized according to a methodology previously described by our group [22]. Tributyrin (TB) was purchased from Sigma-Aldrich (St. Louis, MO, USA). N-hexadecane for 
synthesis was purchased from Merck (Darmstadt, Germany). All solvents used were HPLC grade from Lab-Scan (Dublin, Ireland). The immobilized lipase B from Candida antarctica (Novozym 435) was a gift from Novozymes Spain. Mesoporous silica MS3030 was a kind gift from Silica PQ Corporation (PA, USA).

\subsection{Ethanolysis reaction.}

TB or SCAKG $(1,7 \mathrm{~g})$, ethanol $(5,3 \mathrm{~g})$ and $\mathrm{n}$-hexadecane as internal standard $(0,2 \mathrm{~g})$ were added to a $100 \mathrm{~mL}$ flask and mixed by swirling. Then, the lipase $10 \%$ by weight was added. The flask was placed in an orbital shaker at $50{ }^{\circ} \mathrm{C}$ and $200 \mathrm{rpm}$. Samples of $50 \mu \mathrm{L}$ were withdrawn periodically. The reaction was allowed to proceed for $50 \mathrm{~min}$ to $420 \mathrm{~min}$.

\subsection{Immobilization of Candida antarctica lipase}

The protocol of immobilization of lipase has been previously described [23]. Silica MS 3030 was functionalized by reaction with octyltriethoxysilane in toluene, and the octyl-Silica obtained was used as the carrier.

$1 \mathrm{~mL}$ of ethanol was added for each $100 \mathrm{mg}$ octyl silica and the mixture was left to equilibrate in a closed vial for at least $10 \mathrm{~min}$. $5 \mathrm{~mL}$ of the commercial extract of Candida antarctica lipase B containing 20 mg protein were dissolved in $25 \mathrm{mM}$ phosphate buffer $\mathrm{pH} 7.0$, up to a total volume of 10 $\mathrm{mL}$. After assaying the esterasic activity (hydrolysis of p-nitrophenyl acetate followed spectrophotometrically at $348 \mathrm{~nm}$ ), the enzyme solution was added on the vial containing the silica in ethanol and kept in mild stirring with a helical stirrer. Aliquots from suspension and supernatant were withdrawn at 15 min intervals to assay their respective esterasic activities. Final time was determined by the lack of activity, or low constant activity of the 
supernatant. The suspensions were then filtered and washed three times with $10 \mathrm{~mL}$ volumes of $200 \mathrm{mM}$ phosphate buffer. The supernatants of the immobilization mixtures, as well as the liquids from these washings, were tested for protein desorption by assays of their catalytic activities (esterasic activity). After the last washing, the derivatives were twice suspended in $10 \mathrm{~mL}$ dry acetone, filtered out and finally vacuum dried for at least $30 \mathrm{~min}$ to ensure a complete drying of the catalyst.

\subsection{Analyses of the reaction products by gas chromatography.}

The samples were diluted with chloroform to obtain a final concentration lower than $0.1 \mathrm{mg} / \mathrm{mL}$ for the subsequent analysis by gas chromatography. Separations were performed on a Hewlett-Packard 5890 series II gas chromatograph with on-column injection using a $7 \mathrm{~m} 5 \%$ phenyl methyl silicone capillary column (Quadrex Corporation, New Haven, CT) (0.25 $\mu \mathrm{m}$ i.d). $12 \mathrm{~cm}$ of a $530 \mu \mathrm{m}$ i.d. deactivated column was used as pre-column. Injector and detector temperature was $43^{\circ} \mathrm{C}$ and $360^{\circ} \mathrm{C}$ respectively. The temperature program was as follows: starting at $40{ }^{\circ} \mathrm{C}$ and then heating to $250{ }^{\circ} \mathrm{C}$ at $42^{\circ} \mathrm{C} \mathrm{min}^{-1}$ with $10 \mathrm{~min}$ hold, followed by heating from $250{ }^{\circ} \mathrm{C}$ to $325^{\circ} \mathrm{C}$ at $7.5^{\circ} \mathrm{C}$ min $^{-1}$ with 30 min hold. Helium was used as a carrier gas at a pressure of 5.2 psi. The peaks were computed using GC chemstation software (Agilent Technologies, Santa Clara, CA).

\subsection{Mathematical modeling}

\subsubsection{Reaction rate for the ethanolysis reaction: uniresponse model:}

Rate expressions based on a generalized Michaelis - Menten mechanism for the ethanolysis reaction were utilized (Figure 1). A ping-pong mechanism controlled by the rate of deacylation of the enzyme was proposed. These 
rate expressions are similar to those described by Malcata et al. [24] and by Lessard and Hill [25] for lipase catalyzed hydrolysis reactions. In particular a modification of these rate expressions, presented by Torres and Hill [26], was employed. The main differences with respect to the model proposed by Malcata et al are: 1) the rate expressions contain the concentration of ethanol rather than the concentration of water; 2) a term for the reverse of the ethanolysis reaction is incorporated in the rate expressions. However, in the present study the reverse of the ethanolysis reaction can be neglected based on the findings that nearly $100 \%$ of fatty acid ethyl esters can be achieved in similar systems $[27,28]$. Hence, the rate expression of the model is represented by:

$$
\frac{d[G]}{d t}=\frac{\frac{v_{\max }}{K_{m}}[G][B]}{1+\frac{[G]}{K_{m}}+\frac{[P]}{K_{i}}}
$$

$[G]$ and $[P]$ are the molar concentrations of the parent acylglycerol, and the lower acylglycerol, respectively. $[B]$ denotes the concentration of ethanol, the $v_{\max }$ is the maximum rate of reaction at a particular glyceride bond $[G]$ at a saturating concentration of the substrate (and in the absence of the other glycerides), the $K_{m}$ is the Michaelis-Menten constant for the glycerides and the $K_{i}$ is the inhibition constant for the corresponding ethyl esters. The lumped parameters, $v_{\max }, K_{i}$, and $K_{m}$ are defined as:

$$
\begin{gathered}
v_{\text {max }}=\frac{k_{2} k_{p}\left[E_{T}\right]}{k_{p}+k_{-p}} \\
K_{i}=\frac{k_{3}}{k_{-3}} \\
K_{m}=\frac{k_{-1} k_{-p}}{k_{1}\left(k_{p}+k_{-p}\right)}
\end{gathered}
$$


Eq. (1) can be simplified by reparameterization following the approach of Malcata et al. [24] to yield:

$$
-\frac{d[G]}{d t}=\frac{\Omega_{2}[G][B]}{1+K_{1}[G]}
$$

The corresponding lumped parameters $\left(\Omega_{2}\right.$ and $\left.K_{1}\right)$ are summarized in Table 1.

\subsubsection{Loss of enzyme activity with time}

Knowledge of the rate law governing the deactivation process is often important in modeling enzyme-catalyzed processes. For the purpose of modeling, deactivation is usually treated as if it occurs in a single step. It is often assumed that irreversible deactivation of the active enzyme $(E)$ to an inactive form $\left(E_{d}\right)$ obeys first-order kinetics:

$$
E \stackrel{k_{d}}{\longrightarrow} E_{d}
$$

The manner in which the enzyme activity varies with time is then given by:

$$
a\{t\}=a_{0} \exp \left[-k_{d}\left(t-t_{0}\right)\right]
$$

where $t$ is time; $t_{0}$ is the time at which the first experiment with a particular sample of enzyme is started; $a\{t\}$ is the activity of the enzyme at a particular time $t ; a_{0}$ is the activity of the enzyme at time $t_{0}$; and $k_{d}$ is the first order deactivation rate constant. The parameter $a_{0}$ can arbitrarily be assigned to a value of 1 and subsequent activities are thus normalized with respect to this initial activity.

One of the problems that arise when one tries to evaluate the loss of enzyme activity in batch reactors is the fact that the time of the reaction 
and the time at which the set of experiments was started are equivalent. This fact does not permit one to separate the rate of the reaction from the rate at which the enzyme is losing activity. In order to overcome this problem, data sets were obtained using two cycles of reaction with the same batch of lipase in order to distinguish between the times characteristic of the two types of reaction (ethanolysis and deactivation). Each cycle involves the same elapsed time for the ethanolysis reaction but for one step in the cycle, the elapsed time will differ from the time during which the lipase has been susceptible to deactivation $\left(t-t_{0}\right)$. The mathematical model used in the analysis of the data thus can account for the effect of deactivation of the enzyme in the combined rate expressions for the rates of ethanolysis reaction.

To characterize the loss of enzyme activity, the variable of importance is the time elapsed since the start of the experiments $\left(t-t_{0}\right)$. The amounts of the butyric acid ethyl ester obtained depend not only on the time the reactants in a particular batch are in contact with the lipase, but also the enzymatic activity profile during the time that batch is being processed. Graphical depictions of the resulting functional dependence thus require the use of a three dimensional plot. However, in order to obtain plots that can be more readily interpreted, it is convenient to employ the concept of a pseudo reaction time in order to permit representation of the data in two dimensions [24]. The pseudo reaction time represents a mathematical combination of the time elapsed since the start of the series of experiments and the real reaction time. The pseudo reaction time for a particular batch is defined as 


$$
t^{*}=\frac{\int_{t_{1}}^{t} a\{\hat{t}\} d \hat{t}}{a_{0}}
$$

where $t^{*}$ is the pseudo reaction time, $\hat{t}$ is a dummy variable, $t_{1}$ is the time at which processing of the particular batch of interest was initiated, and $\left(t-t_{1}\right)$ is the (elapsed) time during which a particular batch of oil is in contact with the lipase (the actual reaction time). In physical terms when the rate of deactivation of the enzyme is negligible, the pseudo reaction time $t^{*}$ becomes equal to the actual reaction time $\left(t-t_{1}\right)$. When the rate of deactivation is significant, the difference between the actual reaction time and the pseudo reaction time indicates how much longer the reaction mixture must remain in the reactor to achieve the conversion that would have been achieved if the enzyme had not been partially deactivated. In terms of Equations 7 and 8

$$
t^{*}=\frac{1}{k_{d}}\left\{\left[\exp \left\{-k_{d}\left(t_{1}-t_{0}\right)\right\}\right]-\left[\exp \left\{-k_{d}\left(t-t_{0}\right)\right\}\right]\right\}
$$

Note that as $k_{d}$ approaches zero, the difference in the exponential terms approaches $k_{d}\left(t-t_{1}\right)$ and $t^{*}$ approaches $\left(t-t_{1}\right)$. The reader should also note that the value of $\left(t_{1}-t_{0}\right)$ represents the sum of the times that the enzyme was used as a biocatalyst in previous trials and the total time necessary to recover the catalyst in the various filtration steps prior to its use in the trial of interest.

\section{Results and discussion}

3.1 Direct comparison between lipase-catalyzed ethanolysis of tributyrin and sn-2,3-dibutyroil-1-0-alkylglycerols. 
The weight percentage of ethyl butyrate from the reaction mixtures released during the first 40 minutes of both ethanolysis reactions is shown in Figure 2. It can be observed that the rate of lipase-catalyzed ethanolysis of TB is much faster than that of SCAKG. A higher reaction rate can be expected in the case of tributyrin compared to that of SCAKG because of the fact that TB contains three butyric acid residues and SCAKG only two. However, inspection of the course of the reaction indicates that after 15 minutes of reaction, the percentage of ethyl butyrate released is ca. $10 \%$ and $50 \%$ for ethanolysis of SCAKG and TB respectively. These results indicate that lipase-catalyzed ethanolysis of TB is approximately five times faster than lipase-catalyzed ethanolysis of SCAKG.

\subsection{Time course of the ethanolysis of SCAKG and TB in the presence of}

\section{Novozym 435}

The course of the ethanolysis of SCAKG and TB in the presence of Novozym 435 is depicted in Figure $3 \mathrm{~A}$ and $3 \mathrm{~B}$ respectively. Two trials reutilizing the same batch of lipase were effected in both cases. The differences observed between the two trials assayed for each ethanolysis reaction could be, in part, attributed to partial inactivation of the lipase and were utilized to determine the inactivation constant according to the methodology described in the Mathematical modeling section. Besides the differences in reaction rates between ethanolysis of SCAKG and TB discussed before, it can be observed that monoesterified alkylglycerols reach a plateau after 60 minutes of ethanolysis reaction that it is maintained almost constant during the course of the reaction. This result indicates that at these reaction conditions, Novozym 435 is highly selective for butyric acid residues located at sn-3 position. On the contrary, this plateau is not observed in the case of 
ethanolysis of TB. In this case, 1,3-dibutyrin is rapidly transformed in 2monobutyrin. This observation is consistent with that by Piyatheerawong et al [29].

Inspection of Figures $3 \mathrm{~A}$ and $3 \mathrm{~B}$ again indicates the difference in reaction rates among the two ethanolysis reactions studied. Hence, the percentage of SCAKG and TB after 20 minutes of reaction was ca $50 \%$ and below $1 \%$ respectively.

\subsection{Kinetics modeling of the lipase-catalyzed ethanolysis of SCAKG and TB}

It should be noted that the kinetic model utilized in the present study only includes parameters for the forward reaction and inhibition effects. Hence, to avoid overparametrization, facilitate the non linear regression analysis and based on the findings that nearly $100 \%$ of fatty acid ethyl esters can be achieved in similar ethanolysis reaction systems, Equation 5 does not account for reversibility of the reaction that was considered to be negligible. In this study, in an attempt to improve the results of the ethanolysis reaction towards SCAKG, a second lipase (a lipase from Candida antarctica immobilized on mesoporous silica) was also utilized for the ethanolysis of SCAKG. The kinetic parameters obtained are shown in Table 2.

Inspection of the Table 2 indicates that the parameter corresponding to release of butyric ethyl ester $\left(\Omega_{2}\right)$ is an order of magnitude smaller for ethanolysis of SCAKG than the corresponding for ethanolysis of TB. A higher value of this parameter was attained with the immobilized Candida antarctica on mesoporous silica which indicates that this lipase is slightly faster than Novozym 435 in ethanolysis of SCAKG. 
Negative values for $K_{1}$ were obtained in the three cases under study. Because the lumped parameter $K_{1}$ involves a difference of two terms, namely, $\frac{1}{K_{m}}$ and $\frac{1}{K_{i}}$ (Equation 1 ), negative values can be obtained for $K_{1}$. Regarding the inactivation of lipases the lowest value of $k_{d}$ was obtained for ethanolysis of SCAKG catalyzed by Novozym 435. In this case, the contribution to the fiting of $k_{d}$ was almost negligible and similar results were attained removing this parameter from the model. This result indicates that Candida antarctica lipase is very stable at this reaction conditions, and numerous trials reusing the same batch of lipase should be necessary to get enough data to characterize the activity decay and properly calculate the value of $k_{d}$ for this lipase. For that reason the value reported that corresponds only to two consecutive trials, it is an estimate that should be used for comparison purposes but not to determine the extent of the lipase inactivation. Unfortunately, a higher value of $k_{d}$ was observed for ethanolysis of SCAKG catalyzed by the immobilized Candida antarctica on mesoporous silica.

These results indicate that the immobilization process on Candida antarctica lipase has two opposite effects: 1 ) increases the rate of release of butyric acid ethyl ester (higher value of $\Omega_{2}$ ) and 2) has a deleterious effect on the stability of the biocatalyst (lower value of $k_{d}$ in comparison with that attained with Novozym 435).

In addition, inspection of Table 1 also indicates a relatively high deactivation constant for ethanolysis of TB that can be attributed to an additional 
deleterious effect caused by tributyrin that does not take place in ethanolysis of SCAKG.

The estimated half-life of the lipases under study according to the values of $k_{d}$ attained are 180,35 , and 10 hours for ethanolysis of SCAKG catalyzed by Novozym 435, ethanolysis of SCAKG catalyzed by the immobilized Candida antarctica on mesoporous silica, and ethanolysis of TB catalyzed by Novozym 435 respectively.

\subsection{Kinetic model of the release of ethyl butyrate}

Curves corresponding to fits of kinetics model for release of ethyl butyrate are shown in Figure 4.

The model contains the assumption that deactivation of the enzyme (E) obeys first order kinetics. Hence, the kinetics model corresponds to combination of equation (5) with a first order deactivation term. The reader should also note that the data points correspond to two different trials in which the same enzyme was reused (after recovery from the previous trial). To facilitate inspection of the data, different symbols have been utilized for the data from the two different trials considered in the analysis. The data points corresponding to the same elapsed time within a given trial are characterized by a shift on the pseudo reaction time scale that depends on the extent of inactivation of the lipase. It can be observed that no shift in the pseudo reaction time was observed for the shorter reaction times. The extent of inactivation can only be noticeable at the longer reaction times. Hence, for example for the ethanolysis of SCAKG catalyzed by Candida antarctica lipase immobilized in mesoporous silica (Figure 4B), the reaction time 420 minutes is shifted to a pseudo reaction time of 392 and 340 minutes for the first and the second trial respectively. On the contrary, for 
the ethanolysis of SCAKG catalyzed by Novozym 435 (Figure 4A) no significant shift in the pseudo reaction time is observed at 30 minutes of reaction time for both trials. On the contrary even higher lipase activity is observed at short reaction times which could indicates that other factors (such as recovery of the lipase and experimental errors) could partially interfere with the reported value of $k_{d}$. It should be also indicated that the extent of the inactivation, to be perceptible, should be significantly higher than the experimental error in each trial.

For these reasons (very short reaction times and experimental errors) it is not possible to graphically visualize the extent of inactivation for the ethanolysis of TB catalyzed by Novozym 435.

Inspection of Figure 4A indicates that ethanolysis of SCAKG catalyzed by Novozym 435 occurs in two stages: a first rapid release of ethyl butyrate during the first hour of reaction followed by a second stage with slower release of ethyl butyrate. This effect could be attributed to inhibition effect for accumulation of monoesterified alkylglycerols or ethyl butyrate in the surroundings of the active site of Novozym 435. This phenomenon was not clearly observed neither for ethanolysis of SCAKG catalyzed by Candida antarctica lipase immobilized in mesoporous silica (Figure 4B) nor for ethanolysis of TB catalyzed by Novozym 435 (Figure 4C).

\section{Conclusions}

A very efficient methodology for the ethanolysis of two different triradylglycerols has been attained. Much faster rate of ethanolysis for TB than for SCAKG was observed.

High regio-selectivity of Novozym 435 was observed for the ethanolysis of SCAKG with a rapid accumulation of monoesterified alkylglycerols in the 
reaction mixture that is maintained almost constant the remaining time of the reaction.

The kinetic model developed in this study indicates that the parameter corresponding to release of butyric ethyl ester $\left(\Omega_{2}\right)$ is one order of magnitude higher for the ethanolysis of TB compared to that for the ethanolysis of SCAKG which confirms the experimental observation of the time course of both reactions.

Finally, low inactivation rate was observed for ethanolysis of SCAKG in the presence of Novozym $435\left(k_{d}=3.82 \mathrm{E}-03\right)$. Based on this parameter, the estimated half-life of the lipase is 180 hours, which permit to reutilize the same batch of lipase up to 20 trials.

In conclusion the kinetic model developed in the present study indicates differences in rate of the reaction, selectivity, and provide mechanistic information regarding how these two triradylglycerols are recognized by lipases. In addition, lipase-catalyzed ethanolysis of these two compounds is very useful step for selectively remove fatty acid residues at sn-1, and sn-3 positions and it can be used for subsequent production of structured lipids containing short chain and polyunsaturated fatty acids in a single molecule.

\section{Acknowledgements}

This work was supported by the projects AGL2006-02031/ALI and AGL2008-05655 by Ministerio de Ciencia (Spain) and also by Comunidad Autonoma de Madrid (ALIBIRD, project number S-505/AGR-0153) and Consolider-Ingenio FUN-C-FOOD (CSD2007- 00063). A predoctoral fellowship for Luis Vázquez, provided by Comunidad Autonoma de Madrid, is also acknowledged. 
Table 1. Definitions of model parameters in terms of the intrinsic rate constants.

\begin{tabular}{|c|c|}
\hline Parameter & mechanistic interpretation \\
\hline$\Omega_{2}$ & $\frac{k_{p} k_{1} k_{2}\left[E_{T}\right] / k_{-1} k_{-2}}{\Delta}$ \\
\hline$K_{1}$ & $\frac{1}{\Delta}\left(\frac{k_{1}}{k_{-1}}+\frac{k_{1} k_{2}}{k_{-1} k_{-2}}-\frac{k_{-3}}{k_{3}}\right)$ \\
\hline$\Delta$ & $1+\frac{k_{-3}}{k_{3}}\left[G_{0}\right]$ \\
\hline
\end{tabular}


Table 2. Parameter estimates for the best fits of lipase-catalyzed

ethanolysis of two short chain alkylglycerols and triacylglycerols

\begin{tabular}{llll}
\hline & SCAKG & SCAKG & TB \\
Parameter & Novozym435 & Immobilized $C$. antarctica & Novozym435 \\
\hline$\Omega_{2}$ & $4.73 \mathrm{E}-06 \pm 1.94 \mathrm{E}-06$ & $1.17 \mathrm{E}-05 \pm 1.16 \mathrm{E}-06$ & $3.29 \mathrm{E}-05 \pm 7.22 \mathrm{E}-06$ \\
$K_{1}$ & $-1.38 \mathrm{E}-03 \pm 9.05 \mathrm{E}-08$ & $-1,34 \mathrm{E}-03 \pm 5,08 \mathrm{E}-05$ & $-4.93 \mathrm{E}-04 \pm 1.62 \mathrm{E}-08$ \\
$k_{d}$ & $3.82 \mathrm{E}-03 \pm 1.71 \mathrm{E}-03$ & $2.22 \mathrm{E}-02 \pm 2.66 \mathrm{E}-03$ & $7.60 \mathrm{E}-02 \pm 8.08 \mathrm{E}-03$
\end{tabular}



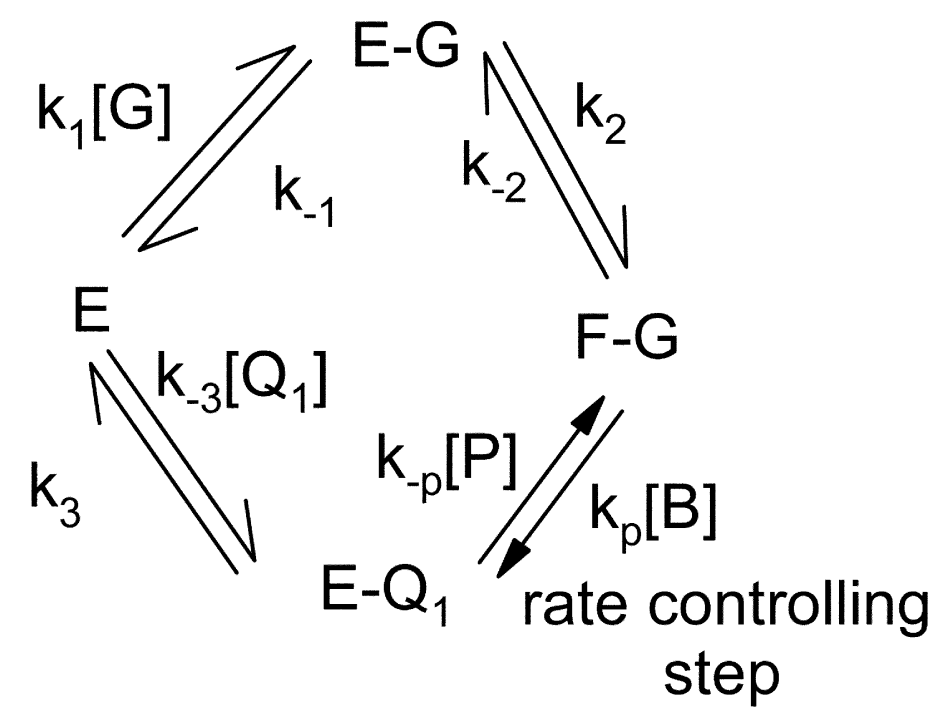


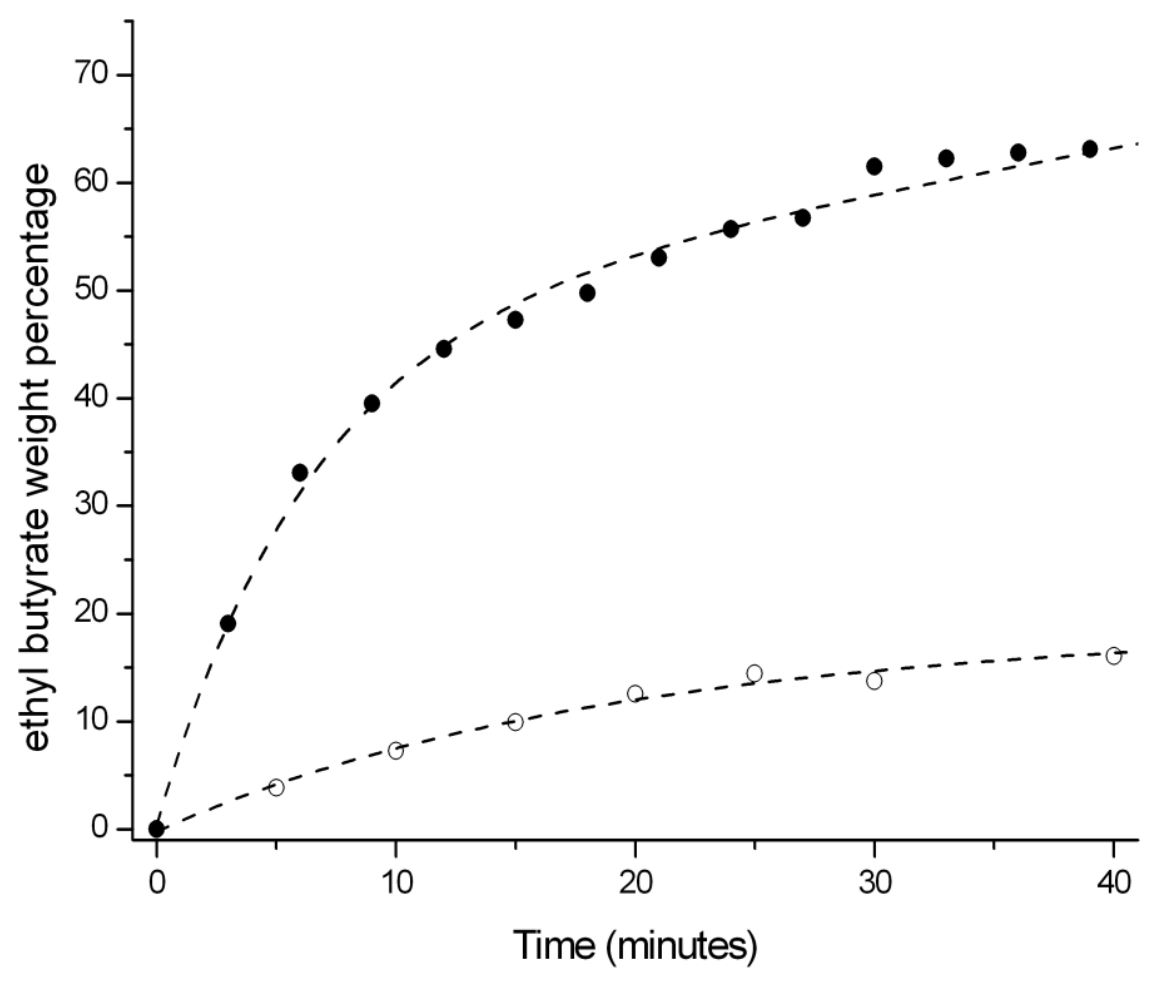



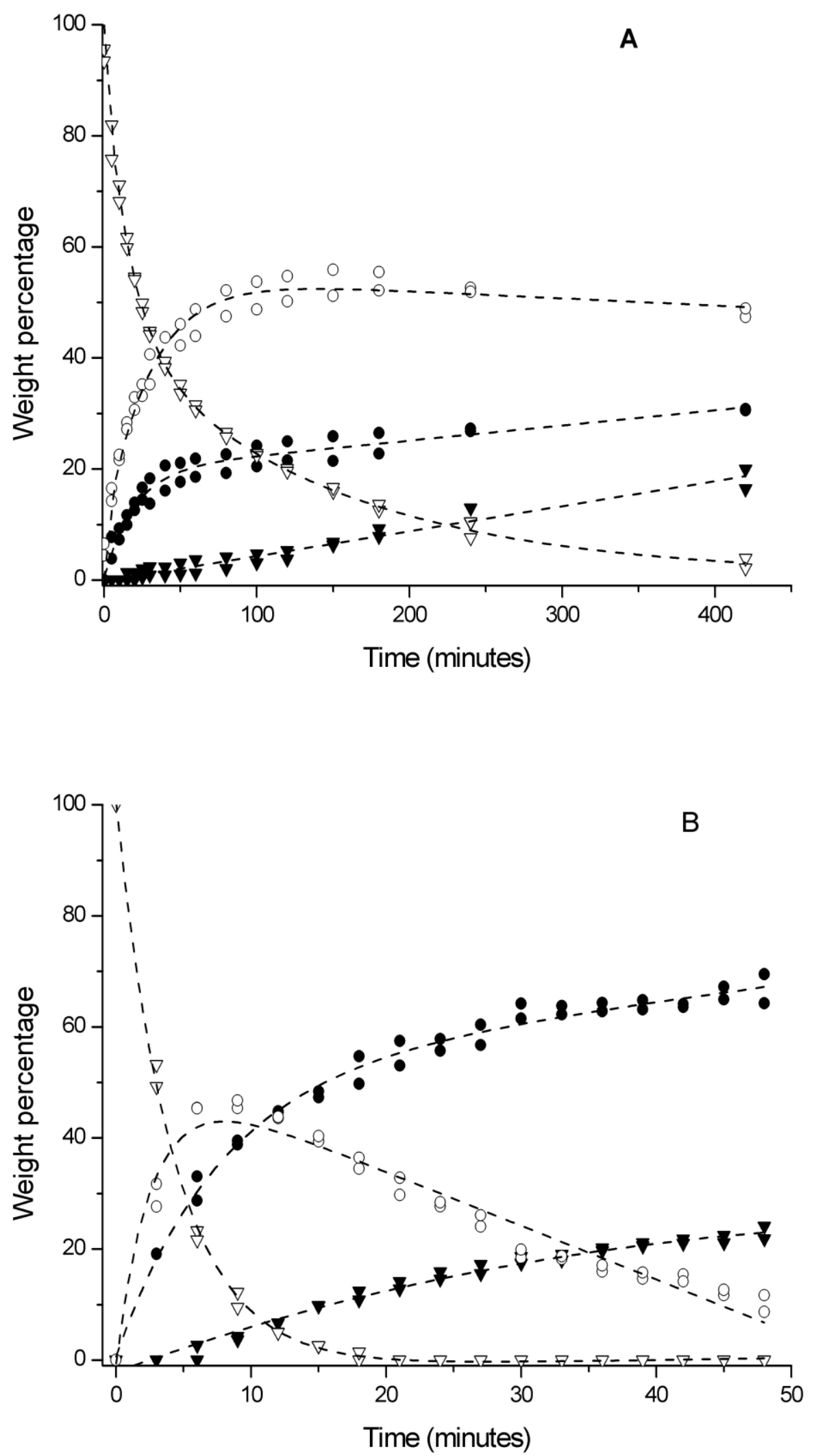

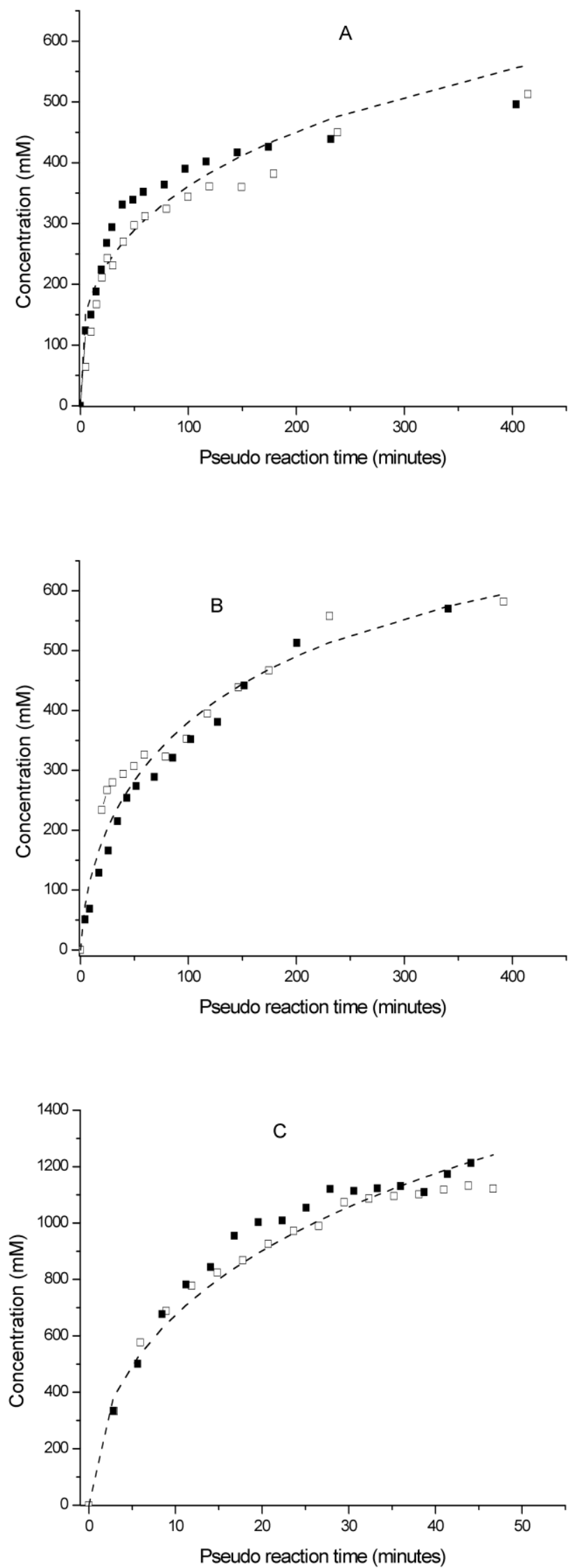


\section{Figure captions}

Figure 1. Schematic representation of the ethanolysis reaction. $E=$ enzyme, $\mathrm{G}=$ glyceryl ester bond (reactant glyceride), $\mathrm{E}-\mathrm{G}=$ activated form of the enzyme and the native ester bond, $\mathrm{F}-\mathrm{G}=$ covalent transformed form of the enzyme and the native ester bond, E-Q = activated form of the enzyme and the fatty acid ethyl ester, $\mathrm{B}=$ ethanol, $\mathrm{P}=$ lower glyceride liberated from the native ester, $Q=$ fatty acid ethyl ester. $k_{1}, k_{-1}, k_{2}, k_{-2}, k_{p}$, $\mathrm{k}_{-\mathrm{p}}, \mathrm{k}_{3}, \mathrm{k}_{-3}$, are rate constants.

Figure 2. Comparison between the rate of release of ethyl butyrate in lipase-catalyzed ethanolysis of tributyrin and short-chain alkyglycerols. filled symbols correspond to tributyrin, Open symbols correspond to short-chain alkyglycerols.

Figure 3. Lipase-catalyzed ethanolysis of short-chain alkyglycerols $(A)$ and tributyrin (B). Figure 3A: open triangles: SCAKG; open circles: monoesterified alkylglycerols, filled circles: ethyl butyrate, filled triangles: nonesterified alkylglycerols. Figure 3B: open triangles: tributyrin, open circles: dibutyrin, filled circles: ethyl butyrate, filled triangles: monobutyrin.

Figure 4. Curves corresponding to fit of kinetics model for lipase-catalyzed ethanolysis of short-chain alkyglycerols in the presence of Novozym 435 (A), in the presence of Candida antarctica immobilized on mesoporous silica

(B) and ethanolysis of tributyrin in the presence of Novozym 435 (C).

Dashed lines correspond to fitted values. Open symbols correspond to trial 1 and filled symbols correspond to trial 2 . 


\section{Bibliography}

[1] H. K.Mangold, in Snyder, FL. (eds). Ether Lipids. Chemistry and biology. New York: Academic, 1972, pp. 157-176.

[2] P. T. Pugliese, K. Jordan, H. Cederberg, J.Brohult, J Altern Complement Med (1998), 4, 87-99.

[3] R. Andreesen, Prog Biochem Pharmacol, (1988), 22, 118-31.

[4] W. E. Berdel, Br J Cancer, (1991) 64, 208-211.

[5] L. Diomede, F Colotta, B Piovani, F Re, E. J. Modest, M. Salmona, Int J Cancer, (1993) 53, 124-130.

[6] J. Palmblad, J. Samuelsson, J. Brohult, Scand J Clin Lab Invest, (1990) $50,363-70$.

[7] K. Hartvigsen, A. Ravandi , R. Harkewicz, H. Kamido, K. Bukhave, G. Hølmer , A. Kuksis, Lipids (2006) 41, 679-693.

[8] A. Tokumura, Prog Lipid Res (1995) 34, 151-184.

[9] A. Toscani, D. R. Soprano, K. J. Soprano, Oncogene Res. (1988), 223238.

[10] L. A. Mickley, S. E. Bates, N. D. Richert, S.Currier, S. Tanaka, F. Foss, N. Rosen, A. T. Fojo, J Biol Chem (1989) 264, 18031-18040.

[11] C. W. Taylor, Y. S. Kim, K. E. Childress-Fields, L. C. Yeoman, Cancer Lett (1992) 62, 95-105.

[12] A.Wachtershauser, S. M. Loitsch, J. Stein, Biochem Biophys Res Commun (2000) 272, 380-385.

[13] A. Hague, G. D. Diaz, D. J. Hicks, S. Krajewski, J. C. Reed, C. Paraskeva, Int J Cancer (1997), 72, 898-905. 
[14] Y.Tanaka, K. K. Bush, T. Eguchi, N. Ikekawa, T. Taguchi, Y. Kobayashi, P. J. Higgins, Arch Biochem Biophys (1990) 276, 415-423.

[15] M. Yoshida, Y. Tanaka, T. Eguchi, N. I. kekawa, N. Saijo, Anticancer Res (1992) 12, 1947-1952.

[16] J. Stein, O. Schröder, V. Milovic, W. F. Caspary, Gastroenterology (1995) 108, 673-679.

[17] O. Schröder, J. Opritz, J. Stein, Digestion (2000) 62, 152-158.

18 J. Stein, M. Zores, O. Schröder, Eur J Nutr (2000) 39, 121-125.

[19] C. Schröder, K. Eckert, H. R. Maurer, Int J Oncol (1998) 13, 13351340.

[20] N. Satya Sree Kolar, R. Barhoumi, J. R. Lupton, R. S. Chapkin, Cancer Res (2007) 67, 5561-5568.

[21] N. Yeevoon, R. Barhoumi, R. B. Tjalkens, Y. Fan, S. Kolar, N. Wang, J. R. Lupton, and R. S.Chapkin, Carcinogenesis (2005) 26, 1914-1921.

[22] C. F. Torres, L. Vazquez, F. J. Señorans, G. Reglero. Process Biochem (2009) 44, 1025-1031.

[23] R. M. Blanco, P. Terreros, N. Muñoz, E. Serra, J Mol Catal B Enzym (2007) 47, 13-20.

[24] F. X. Malcata, C. G. Hill, Jr., C. H. Amundson, Biotech Bioeng (1992) 39, 984-1001.

[25] L. P. Lessard, C. G. Hill, Jr. Biotech Bioeng (2000) 69, 183-195.

[26] C. F. Torres, M. Moeljadi, C.G. Hill, Jr., Biotechnol Bioeng (2003) 83, 274-281.

[27] Y. Yesiloglu. J Am Oil Chem Soc. (2004) 81, 157-160.

[28] Y. Shimada, Y. Watanabe, A. Sugihara, Y. Tominaga, J Mol Catal B Enzym (2002) 17, 133-142. 
[29] W. Piyatheerawong, Y. Iwasaki, T. Yamane, J Chromatogr A (2005) $1068,243-248$. 